\title{
PIECEWISE-MULTILINEAR INTERPOLATION OF A RANDOM FIELD
}

\author{
KONRAD ABRAMOWICZ* AND \\ OLEG SELEZNJEV,$^{* * *}$ Umeå University
}

\begin{abstract}
We consider a piecewise-multilinear interpolation of a continuous random field on a $d$-dimensional cube. The approximation performance is measured using the integrated mean square error. Piecewise-multilinear interpolator is defined by $\mathrm{N}$-field observations on a locations grid (or design). We investigate the class of locally stationary random fields whose local behavior is like a fractional Brownian field, in the mean square sense, and find the asymptotic approximation accuracy for a sequence of designs for large $N$. Moreover, for certain classes of continuous and continuously differentiable fields, we provide the upper bound for the approximation accuracy in the uniform mean square norm.
\end{abstract}

Keywords: Approximation; random field; piecewise-multilinear interpolator; sampling design

2010 Mathematics Subject Classification: Primary 60G60

Secondary 41A63; 41A05

\section{Introduction}

Let a random field $X(t), t \in[0,1]^{d}$, with finite second moment be observed at a finite number of points. Suppose further that the points are vertices of hyperrectangles generated by a grid in a unit hypercube. At any unsampled point we approximate the value of the field by a piecewise-multilinear interpolator, which is a natural extension of a conventional one-dimensional $(d=1)$ piecewise-linear interpolator. For this interpolator, a compact representation and probabilistic interpretation is given. The approximation accuracy is measured by the integrated mean squared error. In this paper we aim to model random fields to a given accuracy based on a finite number of observations. Following Berman (1974), we extend the concept of local stationarity for random fields and focus on fields satisfying this condition. For quadratic mean (q.m.), continuous, locally stationary random fields, we derive the exact asymptotic behavior of the approximation error. A method is proposed for determining the asymptotically optimal knot (sample points) distribution between the mesh dimensions. We also study optimality of knot allocation along coordinates of the sampling grid. Additionally, for q.m. continuous and continuously differentiable fields satisfying Hölder-type conditions, we determine asymptotic upper bounds for the approximation accuracy.

The problem of random field approximation arises in many research and applied areas, such as Gaussian random fields modeling (see Adler and Taylor (2007) and Brouste et al. (2007)), environmental and geosciences (see Christakos (1992) and Stein (1999)), sensor networks (see Zhang and Wicker (2005)), and image processing (see Pratt (2007)). The upper bound

Received 17 February 2011; revision received 4 February 2013.

* Postal address: Department of Mathematics and Mathematical Statistics, Umeå University, SE-901 87 Umeå, Sweden.

** Email address: oleg.seleznjev@math.umu.se 
for the approximation error for isotropic random fields satisfying Hölder-type conditions is given in Ritter et al. (1995). Müller-Gronbach (1998) considered affine linear approximation methods and hyperbolic cross designs for fields with a covariance function of tensor type. An optimal allocation of the observations for Gaussian random fields with product-type kernel is investigated in Müller-Gronbach and Schwabe (1996). Su (1997) studied limit behavior of the piecewise-constant estimator for random fields with a particular form of covariance function. Benhenni (2001) investigated exact asymptotics of a stationary spatial process approximation based on an equidistant sampling. The approximation complexity and the curse of dimensionality for additive random fields are broadly discussed in Lifshits and Zani (2008). In the one-dimensional case, the piecewise-linear interpolation of continuous stochastic processes is considered in, e.g. Seleznjev (1996). Results for the approximation of locally stationary processes can be found in, e.g. Seleznjev (2000), Hüsler et al. (2003), and Abramowicz and Seleznjev (2011). Ritter (2000) contains a very detailed survey of various random process and field approximation problems. For an extensive study of approximation problems in the deterministic setting, we refer the reader to, e.g. Nikolskii (1975), de Boor et al. (2008), and Kuo et al. (2009).

The paper is organized as follows. First we introduce the basic notation. In Section 2, we consider a piecewise-multilinear approximation of locally stationary random fields. We derive exact asymptotics and a formula for the optimal interdimensional knot distribution. In the second part of this section, we provide an asymptotic upper bound for the approximation accuracy for q.m. continuous and differentiable fields satisfying Hölder-type conditions. In Section 3, we present the results of numerical experiments, while Section 4 contains the proofs of the statements from Section 2.

\subsection{Basic notation}

Let $X=X(t), t \in \mathscr{D}:=[0,1]^{d}$, be a real-valued random field defined on a probability space $(\Omega, \mathcal{F}, \mathbb{P})$. Assume that, for every $\boldsymbol{t}$, the random variable $X(\boldsymbol{t})$ lies in the normed linear space $L^{2}(\Omega)=L^{2}(\Omega, \mathcal{F}, \mathbb{P})$ of random variables with finite second moment and identified equivalent elements with respect to $\mathbb{P}$. We set $\|\xi\|:=\left(\mathbb{E}\left(\xi^{2}\right)\right)^{1 / 2}$ for all $\xi \in L^{2}(\Omega)$ and consider the approximation based on the normed linear spaces of q.m. continuous and continuously differentiable random fields denoted by $\mathcal{C}(\mathscr{D})$ and $\mathcal{C}^{1}(\mathscr{D})$, respectively. We define the following norms for any $X \in \mathcal{C}(\mathscr{D})$ by setting

$$
\|X\|_{2}:=\left(\int_{\mathscr{D}}\|X(\boldsymbol{t})\|^{2} \mathrm{~d} \boldsymbol{t}\right)^{1 / 2}, \quad\|X\|_{\infty}:=\max _{\boldsymbol{t} \in \mathcal{D}}\|X(\boldsymbol{t})\| .
$$

For $k \leq d$, let $\boldsymbol{l}=\left(l_{1}, \ldots, l_{k}\right)$ be a vector of positive integers such that $\sum_{j=1}^{k} l_{j}=d$, and let $L_{i}:=\sum_{j=1}^{i} l_{j}, i=0, \ldots k, L_{0}=0$, be the sequence of its cumulative sums. Then the vector $\boldsymbol{l}$ defines the $\boldsymbol{l}$-decomposition of $\mathscr{D}$ into $\mathscr{D}^{1} \times \mathscr{D}^{2} \times \cdots \times \mathscr{D}^{k}$, with the $l_{j}$-cube $\mathscr{D}^{j}:=[0,1]^{l_{j}}, j=1, \ldots, k$. For any $s \in \mathscr{D}$, we denote the coordinates vector corresponding to the $j$ th component of the decomposition by

$$
\boldsymbol{s}^{j}=\boldsymbol{s}^{j}(\boldsymbol{l}):=\left(s_{L_{j-1}+1}, \ldots, s_{L_{j}}\right) \in \mathcal{D}^{j}, \quad j=1, \ldots, k .
$$

For a vector $\boldsymbol{\alpha}=\left(\alpha_{1}, \ldots, \alpha_{k}\right), 0<\alpha_{j}<2, j=1, \ldots, k$, and the decomposition vector $\boldsymbol{l}=\left(l_{1}, \ldots, l_{k}\right)$, we define

$$
|\boldsymbol{s}|_{\boldsymbol{\alpha}}:=\sum_{j=1}^{k}\left|\boldsymbol{s}^{j}\right|^{\alpha_{j}} \quad \text { for all } \boldsymbol{s}=\left(\boldsymbol{s}^{1}, \ldots, \boldsymbol{s}^{k}\right) \in \mathscr{D}
$$


with the Euclidean norms $\left|\boldsymbol{s}^{j}\right|, \boldsymbol{s}^{j} \in \mathscr{D}^{j}, j=1, \ldots, k$. Similarly, for any $\boldsymbol{r} \in \mathbb{R}^{d}$, let $\boldsymbol{r}=\left(\boldsymbol{r}^{1}, \ldots, \boldsymbol{r}^{k}\right)$ with the $j$ th component $\boldsymbol{r}^{j} \in \mathbb{R}^{l_{j}}, j=1, \ldots, k$.

\subsection{Classes of random fields}

Now we introduce the classes of random fields considered in this paper.

Definition 1. Let $X \in \mathcal{C}(\mathscr{D})$. For fixed vectors $\boldsymbol{\alpha}$ and $\boldsymbol{l}$, and a positive constant $C$, we define the class $\mathcal{C}_{l}^{\alpha}(\mathscr{D}, C)$ of random fields satisfying Hölder's condition, and say that $X \in \mathcal{C}_{l}^{\alpha}(\mathscr{D}, C)$ if

$$
\|X(t+s)-X(t)\|^{2} \leq C|s|_{\alpha} \text { for all } t, t+s \in \mathcal{D} .
$$

Definition 2. Let $X \in \mathcal{C}(\mathscr{D})$. For fixed vectors $\boldsymbol{\alpha}$ and $\boldsymbol{l}$, and a vector function $c(\boldsymbol{t})=$ $\left(c_{1}(\boldsymbol{t}), \ldots, c_{k}(\boldsymbol{t})\right), \boldsymbol{t} \in \mathscr{D}$, with positive and continuous functions $c_{1}(\cdot), \ldots, c_{k}(\cdot)$, we define the class $\mathscr{B}_{l}^{\alpha}(\mathscr{D}, c(\cdot))$ of locally stationary random fields, and write $X \in \mathscr{B}_{l}^{\alpha}(\mathscr{D}, c(\cdot))$ if

$$
\frac{\|X(\boldsymbol{t}+\boldsymbol{s})-X(\boldsymbol{t})\|^{2}}{\sum_{j=1}^{k} c_{j}(\boldsymbol{t})\left|\boldsymbol{s}^{j}\right|^{\alpha_{j}}} \rightarrow 1 \quad \text { as } \boldsymbol{s} \rightarrow 0 \text { uniformly in } \boldsymbol{t} \in \mathscr{D} \text {. }
$$

We assume additionally that, for $j=1, \ldots, k$, the function $c_{j}(\cdot)$ is invariant with respect to permutations of coordinates within the $j$ th component.

For the classes $\mathcal{C}_{l}^{\alpha}$ and $\mathcal{B}_{l}^{\alpha}$, the within-component smoothness is defined by the vector $\boldsymbol{\alpha}=\left(\alpha_{1}, \ldots, \alpha_{k}\right)$. We denote the vector describing the smoothness for each coordinate by $\boldsymbol{\alpha}^{*}=\left(\alpha_{1}^{*}, \ldots, \alpha_{d}^{*}\right)$, where $\alpha_{i}^{*}=\alpha_{j}, i=L_{j-1}+1, \ldots, L_{j}, j=1, \ldots, k$.

Example 1. Let $\boldsymbol{m}=\left(m_{1}, \ldots, m_{k}\right)$ be a decomposition vector of $[0,1]^{m}$, and let $m=$ $\sum_{j=1}^{k} m_{j}$. Denote by $B_{\boldsymbol{\beta}, \boldsymbol{m}}(\boldsymbol{t})$ for $\boldsymbol{t} \in[0,1]^{m}$ and $\boldsymbol{\beta}=\left(\beta_{1}, \ldots, \beta_{k}\right), 0<\beta_{j}<2$, $j=1, \ldots, k$, an $m$-dimensional fractional Brownian field (FBF) with covariance function $r(\boldsymbol{t}, \boldsymbol{s})=\frac{1}{2}\left(|\boldsymbol{t}|_{\boldsymbol{\beta}}+|\boldsymbol{s}|_{\boldsymbol{\beta}}-|\boldsymbol{t}-\boldsymbol{s}|_{\boldsymbol{\beta}}\right)$. Then $B_{\boldsymbol{\beta}, \boldsymbol{m}}$ has stationary increments,

$$
\left\|B_{\beta, m}(t+s)-B_{\beta, m}(t)\right\|^{2}=|s|_{\beta}, \quad t, t+s \in[0,1]^{m},
$$

and, therefore, $B_{\boldsymbol{\beta}, \boldsymbol{m}} \in \mathcal{B}_{\boldsymbol{m}}^{\boldsymbol{\beta}}\left([0,1]^{m}, c(\cdot)\right)$ with local stationarity functions $c_{1}(\boldsymbol{t})=\cdots=$ $c_{k}(\boldsymbol{t})=1, \boldsymbol{t} \in[0,1]^{m}$. In particular, if $k=1$ then $B_{\beta, m}(\boldsymbol{t})$ for $\boldsymbol{t} \in[0,1]^{m}, 0<\beta<2$ and $m \in \mathbb{N}$, is an FBF with covariance function

$$
r(\boldsymbol{t}, \boldsymbol{s})=\frac{1}{2}\left(|\boldsymbol{t}|^{\beta}+|\boldsymbol{s}|^{\beta}-|\boldsymbol{t}-\boldsymbol{s}|^{\beta}\right), \quad \boldsymbol{t}, \boldsymbol{s} \in[0,1]^{m} .
$$

For $X \in \mathcal{C}^{1}(\mathscr{D})$, let $X_{j}^{\prime}(t), t \in \mathscr{D}$, denote a q.m. partial derivative of $X$ with respect to the $j$ th coordinate.

Definition 3. Let $X \in \mathcal{C}^{1}(\mathscr{D})$. For a fixed vector $\boldsymbol{\alpha}^{*}=\left(\alpha_{1}^{*}, \ldots, \alpha_{d}^{*}\right)$ and a positive constant $C$, we define the class of random fields $\mathcal{C}^{1, \alpha^{*}}(\mathscr{D}, C)$, and say that $X \in \mathcal{C}^{1, \alpha^{*}}(\mathscr{D}, C)$ if, for all $t, t+s \in \mathcal{D}$

$$
\left\|X_{j}^{\prime}\left(t_{1}, \ldots, t_{j}+s_{j}, \ldots, t_{d}\right)-X_{j}^{\prime}\left(t_{1}, \ldots, t_{j}, \ldots, t_{d}\right)\right\|^{2} \leq C\left|s_{j}\right|^{\alpha_{j}^{*}}, \quad j=1, \ldots, d .
$$

Moreover, we say that $X \in \mathcal{C}_{l}^{1, \alpha}(\mathscr{D}, C)$ with $\boldsymbol{\alpha}=\left(\alpha_{1}, \ldots, \alpha_{k}\right)$ if $X \in \mathcal{C}^{1, \alpha^{*}}(\mathscr{D}, C)$ and, for a given partition vector $l, \alpha_{i}:=\alpha_{L_{i-1}+1}^{*}=\cdots=\alpha_{L_{i}}^{*}, i=1, \ldots, k$. 


\subsection{Cross regular designs}

Let $X$ be sampled at $N$ distinct design (grid) points $T_{N}:=\left\{\boldsymbol{t}_{\boldsymbol{i}}=\left(t_{1, i_{1}}, \ldots, t_{d, i_{d}}\right): \boldsymbol{i}=\right.$ $\left.\left(i_{1}, \ldots, i_{j}\right), 0 \leq i_{j} \leq n_{j}^{*}, j=1, \ldots, d\right\}$, where

$$
\prod_{j=1}^{d}\left(n_{j}^{*}(N)+1\right)=N .
$$

Since optimal designs for a fixed $N$ are difficult to construct, for asymptotic results, we develop the approach introduced by Sacks and Ylvisaker (1966) for some time series models (for approximation problems, see, for example, Su and Cambanis (1993) and Seleznjev (2000)). For $s \in[0,1], j=1, \ldots, d$, let $h_{j}^{*}(s)$ be positive and continuous density functions, called within-dimensional densities, and

$$
h^{*}(\boldsymbol{t}):=\left(h_{1}^{*}\left(t_{1}\right), \ldots, h_{d}^{*}\left(t_{d}\right)\right), \quad \boldsymbol{t} \in[0,1]^{d} .
$$

We define the inter-dimensional knot distribution determined by a vector function

$$
\pi^{*}(N):=\left(n_{1}^{*}(N), \ldots, n_{d}^{*}(N)\right), \quad N \in \mathbb{N},
$$

where $n_{j}^{*}(N) \in \mathbb{N}, \lim _{N \rightarrow \infty} n_{j}^{*}(N)=\infty, j=1, \ldots, d$, and (5) holds. We suppress the argument $N$ for the sampling grid sizes $n_{j}^{*}=n_{j}^{*}(N), j=1, \ldots, d$, when doing so causes no confusion.

Definition 4. (Cross regular designs.) For functions $h^{*}(\cdot)$ and $\pi^{*}(\cdot)$, the corresponding cross regular sequence of sampling designs $T_{N}:=\left\{\boldsymbol{t}_{\boldsymbol{i}}=\left(t_{1, i_{1}}, \ldots, t_{d, i_{d}}\right): \boldsymbol{i}=\left(i_{1}, \ldots, i_{d}\right)\right.$, $\left.0 \leq i_{j} \leq n_{j}^{*}, j=1, \ldots, d\right\}$ is generated by the equations

$$
\int_{0}^{t_{j, i}} h_{j}^{*}(v) \mathrm{d} v=\frac{i}{n_{j}^{*}}, \quad i=0,1, \ldots, n_{j}^{*}, j=1, \ldots, d .
$$

The introduced classes of random fields have the same smoothness and local behavior for each coordinate of the components generated by a decomposition vector $l$. Therefore, in the following, we use only approximation designs with the same within- and inter-dimensional knot distributions within the components. Formally, for the partition generated by the vector $\boldsymbol{l}=\left(l_{1}, \ldots, l_{k}\right)$, we consider cross regular sequences of designs $T_{N}$, defined by the vector functions $h:=\left(h_{1}, \ldots, h_{k}\right)$ and $\pi(N):=\left(n_{1}(N), \ldots, n_{k}(N)\right)$, as follows:

$$
h_{i}^{*}(\cdot) \equiv h_{j}(\cdot), \quad n_{i}^{*}=n_{j}, \quad i=L_{j-1}+1, \ldots, L_{j}, j=1, \ldots, k .
$$

We call the functions $h_{1}(\cdot), \ldots, h_{k}(\cdot)$ and $\pi(\cdot)$ within-component densities and inter-component knot distribution, respectively. The corresponding property of the sequence of designs $T_{N}$ is denoted by: $T_{N}$ is $c R S(h, \pi, l)$.

\subsection{Piecewise-multilinear interpolator}

For a given cross regular sequence of sampling designs $T_{N}$, the hypercube $\mathscr{D}$ is partitioned into $M=\prod_{j=1}^{d} n_{j}^{*}$ disjoint hyperrectangles $\mathscr{D}_{i}$, where $\boldsymbol{i}=\left(i_{1}, \ldots, i_{d}\right), 0 \leq i_{j} \leq n_{j}^{*}-1$, and $j=1, \ldots, d$. Let $\mathbf{1}_{d}=(1, \ldots, 1)$ denote a $d$-dimensional vector of $1 \mathrm{~s}$. The hyperrectangle $\mathscr{D}_{\boldsymbol{i}}$ is determined by the vertex $\boldsymbol{t}_{i}=\left(t_{1, i_{1}}, \ldots, t_{j, i_{d}}\right)$ and the main diagonal $\boldsymbol{r}_{\boldsymbol{i}}=\boldsymbol{t}_{\boldsymbol{i}+\mathbf{1}_{d}}-\boldsymbol{t}_{i}$, i.e.

$$
\mathscr{D}_{\boldsymbol{i}}:=\left\{\boldsymbol{t}: \boldsymbol{t}=\boldsymbol{t}_{\boldsymbol{i}}+\boldsymbol{r}_{\boldsymbol{i}} * \boldsymbol{s}, \boldsymbol{s}=\left(s_{1}, \ldots, s_{d}\right) \in[0,1]^{d}\right\},
$$


where ' $*$ ' denotes the coordinatewise multiplication, i.e. for $\boldsymbol{x}=\left(x_{1}, \ldots, x_{d}\right)$ and $\boldsymbol{y}=$ $\left(y_{1}, \ldots, y_{d}\right), \boldsymbol{x} * \boldsymbol{y}:=\left(x_{1} y_{1}, \ldots, x_{d} y_{d}\right)$. For a random field $X \in \mathcal{C}(\mathscr{D})$, define a piecewisemultilinear interpolator (PMI) with knots $T_{N}$ by

$$
X_{N}(\boldsymbol{t}):=X_{N}\left(X, T_{N}\right)(\boldsymbol{t})=\mathbb{E}_{\boldsymbol{\eta}} X\left(\boldsymbol{t}_{i}+\boldsymbol{r}_{\boldsymbol{i}} * \boldsymbol{\eta}\right), \quad \boldsymbol{t} \in \mathscr{D}_{\boldsymbol{i}}, \boldsymbol{t}=\boldsymbol{t}_{i}+\boldsymbol{r}_{i} * \boldsymbol{s},
$$

where $\boldsymbol{\eta}=\left(\eta_{1}, \ldots, \eta_{d}\right)$ and $\eta_{1}, \ldots, \eta_{d}$ are auxiliary independent Bernoulli random variables with means $s_{1}, \ldots, s_{d}$, respectively, i.e. $\eta_{j} \in \operatorname{Be}\left(s_{j}\right), j=1, \ldots, d$. This interpolator is continuous and piecewise linear along all coordinates.

Example 2. Let $d=2, N=4$, and $\mathcal{D}=[0,1]^{2}$. Then $\boldsymbol{t}=\boldsymbol{s}, \boldsymbol{r}=(1,1)$,

$$
\begin{aligned}
X_{N}(\boldsymbol{t}) & =\mathbb{E}_{\boldsymbol{\eta}} X(\boldsymbol{\eta}) \\
& =X(0,0)\left(1-t_{1}\right)\left(1-t_{2}\right)+X(1,0) t_{1}\left(1-t_{2}\right)+X(0,1)\left(1-t_{1}\right) t_{2}+X(1,1) t_{1} t_{2},
\end{aligned}
$$

and $X_{N}$ is a conventional bilinear interpolator (see, e.g. Lancaster and Šalkauskas (1986)).

In this article, we consider the accuracy of the approximation to $X$ by $X_{N}$ with respect to the integrated mean-squared error (IMSE)

$$
e_{N}^{2}=e_{N}(X, h, \pi, l)^{2}:=\left\|X-X_{N}\right\|_{2}^{2}:=\int_{\mathscr{D}} e_{N}(\boldsymbol{t})^{2} \mathrm{~d} \boldsymbol{t}, \quad e_{N}(\boldsymbol{t})^{2}:=\left\|X(\boldsymbol{t})-X_{N}(\boldsymbol{t})\right\|^{2} .
$$

We introduce some additional notation used throughout the paper. For sequences of real numbers $u_{n}$ and $v_{n}$, we write $u_{n} \lesssim v_{n}$ if $\lim _{n \rightarrow \infty} u_{n} / v_{n} \leq 1, u_{n} \sim v_{n}$ if $\lim _{n \rightarrow \infty} u_{n} / v_{n}=1$, and $u_{n} \asymp v_{n}$ if there exist positive constants $c_{1}$ and $c_{2}$ such that $c_{1} u_{n} \leq v_{n} \leq c_{2} u_{n}$ for large enough $n$.

\section{Results}

Let $B_{\beta, m}(\boldsymbol{t})$ for $\boldsymbol{t} \in \mathbb{R}_{+}^{m}, 0<\beta<2$, and $m \in \mathbb{N}$ denote an FBF with covariance function (3). For any $\boldsymbol{u} \in \mathbb{R}_{+}^{m}$, we define

$$
b_{\beta, m}(\boldsymbol{u}):=\int_{[0,1]^{m}}\left\|B_{\beta, m}(\boldsymbol{u} * \boldsymbol{s})-\mathbb{E}_{\boldsymbol{\eta}} B_{\beta, m}(\boldsymbol{u} * \boldsymbol{\eta})\right\|^{2} \mathrm{~d} \boldsymbol{s},
$$

where $\boldsymbol{\eta}=\left(\eta_{1}, \ldots, \eta_{m}\right)$ and $\eta_{1}, \ldots, \eta_{m}$ are independent Bernoulli random variables $\eta_{j} \in \operatorname{Be}\left(s_{j}\right), j=1, \ldots, m$. Then $b_{\beta, m}(\boldsymbol{u})$ is the IMSE of approximation for $B_{\beta, m}(\boldsymbol{u} * \boldsymbol{t})$, $t \in[0,1]^{m}$, by the PMI with $2^{m}$ observations of the vertices of the unit hypercube.

Consider first the interpolation of a random field $X$ by the PMI $I(\boldsymbol{t})=I(X, \boldsymbol{\tau}, \boldsymbol{r}, t)$ on a small hyperrectangle $\mathcal{K}=\mathcal{K}(\boldsymbol{\tau}, r):=\left\{\boldsymbol{t}: \boldsymbol{t}=\boldsymbol{\tau}+\boldsymbol{r} * \boldsymbol{s}, \boldsymbol{s}=\left(s_{1}, \ldots, s_{d}\right) \in[0,1]^{d}\right\}$, $\mathcal{K} \subset \mathscr{D}$, with observations of the vertices of $\mathcal{K}$. Let the corresponding IMSE

$$
e(\boldsymbol{\tau}, \boldsymbol{r})^{2}:=\|X-I(X, \boldsymbol{\tau}, \boldsymbol{r})\|_{2}^{2}=\int_{\mathcal{K}} e(\boldsymbol{\tau}, \boldsymbol{r}, \boldsymbol{t})^{2} \mathrm{~d} \boldsymbol{t},
$$

with the mean-squared error $e(\boldsymbol{\tau}, \boldsymbol{r}, \boldsymbol{t})^{2}:=\|X(\boldsymbol{t})-I(X, \boldsymbol{\tau}, \boldsymbol{r}, t)\|^{2}$. For the mean-squared error $e(\tau, \boldsymbol{r}, \boldsymbol{t})^{2}$, by the Cauchy-Schwarz inequality, we obtain

$$
\begin{aligned}
e_{N}(\boldsymbol{\tau}, \boldsymbol{r}, \boldsymbol{t})^{2} & =\mathbb{E}\left(\mathbb{E}_{\boldsymbol{\eta}}(X(\boldsymbol{\tau}+\boldsymbol{r} * \boldsymbol{s})-X(\boldsymbol{\tau}+\boldsymbol{r} * \boldsymbol{\eta}))\right)^{2} \\
& \leq \mathbb{E}\left(\mathbb{E}_{\boldsymbol{\eta}}(X(\boldsymbol{\tau}+\boldsymbol{r} * \boldsymbol{s})-X(\boldsymbol{\tau}+\boldsymbol{r} * \boldsymbol{\eta}))^{2}\right) \\
& =\mathbb{E}_{\boldsymbol{\eta}}\|X(\boldsymbol{\tau}+\boldsymbol{r} * \boldsymbol{s})-X(\boldsymbol{\tau}+\boldsymbol{r} * \boldsymbol{\eta})\|^{2} .
\end{aligned}
$$

Denote by $V(\mathcal{K})$ the volume of the hyperrectangle $\mathcal{K}$. 
Lemma 1. Let $X \in \mathcal{B}_{l}^{\alpha}(\mathcal{D}, c(\cdot))$ be a locally stationary random field approximated on $\mathcal{K} \subset \mathbb{D}$ by the PMI I $(\boldsymbol{t})=I(X, \boldsymbol{\tau}, \boldsymbol{r}, t)$. Then

$$
e(\boldsymbol{\tau}, \boldsymbol{r})^{2}=\left(\sum_{j=1}^{k} c_{j}(\boldsymbol{\tau}) b_{\alpha_{j}, l_{j}}\left(\boldsymbol{r}^{j}\right)\right) V(\mathcal{K})(1+\varepsilon(\boldsymbol{\tau}, \boldsymbol{r})),
$$

where $\varepsilon(\boldsymbol{r}):=\max \{|\varepsilon(\boldsymbol{\tau}, \boldsymbol{r})|, \boldsymbol{\tau} \in \mathscr{D}\}=\mathrm{o}(1)$ as $|\boldsymbol{r}| \rightarrow 0$.

In the following theorem, we provide an exact asymptotic for the IMSE $e_{N}^{2}$ of a PMI approximation of a local stationary field when a cross regular sequence of sampling designs is used.

Theorem 1. Let $X \in \mathscr{B}_{l}^{\alpha}(\mathscr{D}, c(\cdot))$ be a locally stationary random field approximated by the PMI $X_{N}\left(X, T_{N}\right)$, where $T_{N}$ is $c R S(h, \pi, l)$. Then

$$
\left\|X-X_{N}\right\|_{2}^{2} \sim \sum_{j=1}^{k} \frac{v_{j}}{n_{j}^{\alpha_{j}}}>0 \quad \text { as } N \rightarrow \infty,
$$

where

$$
v_{j}:=\int_{\mathscr{D}} c_{j}(\boldsymbol{t}) b_{\alpha_{j}, l_{j}}\left(H_{j}\left(\boldsymbol{t}^{j}\right)\right) \mathrm{d} \boldsymbol{t}>0,
$$

and $H_{j}\left(\boldsymbol{t}^{j}\right):=\left(1 / h_{j}\left(t_{L_{j-1}+1}\right), \ldots, 1 / h_{j}\left(t_{L_{j}}\right)\right), j=1, \ldots, k$.

Remark 1. If, for the $j$ th component, the uniform within-component knot distribution is used, i.e. $h_{j}(s)=1, s \in[0,1]$, then the asymptotic constant is reduced to

$$
v_{j}=\tilde{b}_{\alpha_{j}, l_{j}} \int_{\mathscr{D}} c_{j}(\boldsymbol{t}) \mathrm{d} \boldsymbol{t},
$$

where $\tilde{b}_{\alpha_{j}, l_{j}}:=b_{\alpha_{j}, l_{j}}\left(\mathbf{1}_{l_{j}}\right), \mathbf{1}_{l_{j}}:=(1, \ldots, 1) \in \mathbb{R}^{l_{j}}, j=1, \ldots, k$.

In Theorem 1, the approximation accuracy is determined by the sampling grid sizes $n_{j}$. The next theorem provides the asymptotically optimal inter-component knot distribution for a given total number of observation points $N$. Define

$$
\rho:=\left(\sum_{i=1}^{k} \frac{l_{i}}{\alpha_{i}}\right)^{-1}=\left(\sum_{i=1}^{d} \frac{1}{\alpha_{i}^{*}}\right)^{-1}, \quad \kappa:=\prod_{j=1}^{k} v_{j}^{l_{j} / \alpha_{j}},
$$

where $d \cdot \rho$ is the harmonic mean of the smoothness parameters $\alpha_{j}^{*}, j=1, \ldots, d$.

Theorem 2. Let $X \in \mathcal{B}_{l}^{\alpha}(D, c(\cdot))$ be a locally stationary random field approximated by the PMI $X_{N}\left(X, T_{N}\right)$, where $T_{N}$ is cRS $(h, \pi, l)$ with $\boldsymbol{l}=\left(l_{1}, \ldots, l_{k}\right)$. Then

$$
\left\|X-X_{N}\right\|_{2}^{2} \gtrsim k \frac{\kappa^{\rho}}{N^{\rho}} \quad \text { as } N \rightarrow \infty .
$$

Moreover, for the asymptotically optimal inter-component knot allocation $\pi_{\mathrm{opt}}=\left(n_{1, \mathrm{opt}}, \ldots\right.$, $\left.n_{k, \mathrm{opt}}\right)$ with

$$
n_{j, \mathrm{opt}} \sim \frac{N^{\rho / \alpha_{j}} v_{j}^{1 / \alpha_{j}}}{\kappa^{\rho / \alpha_{j}}} \text { as } N \rightarrow \infty, j=1, \ldots, k,
$$

the equality in (9) is attained asymptotically. 
The above result agrees with the intuition that more points should be distributed in directions with lower smoothness parameters. Note that the optimal inter-component knot distribution leads to an increased approximation rate.

Remark 2. Let $X \in \mathscr{B}_{l}^{\alpha}(\mathscr{D}, c(\cdot))$ with $k=d$ and $\alpha_{i} \neq \alpha_{j}$ for some $i, j=1, \ldots, d$, and $\underline{\alpha}:=\min _{i=1, \ldots, d} \alpha_{i}$, i.e. $\rho>\underline{\alpha} / d$. Consider the approximation errors $e_{N}\left(\pi_{\mathrm{uni}}\right)$ and $e_{N}\left(\pi_{\mathrm{opt}}\right)$ corresponding to the uniform inter-component knot distribution, $n_{1}=\cdots=n_{d} \sim N^{1 / d}$, and the sampling distribution (10), respectively. Then by Theorems 1 and 2 we have

$$
\frac{e_{N}\left(\pi_{\mathrm{opt}}\right)}{e_{N}\left(\pi_{\mathrm{uni}}\right)} \asymp \frac{N^{\underline{\alpha} /(2 d)}}{N^{\rho / 2}}=\frac{1}{N^{\gamma}} \quad \text { as } N \rightarrow \infty, \quad \gamma:=\frac{\rho}{2}-\frac{\underline{\alpha}}{2 d}>0 .
$$

Example 3. Let $d=k=2, \alpha_{1}=\frac{2}{3}$, and $\alpha_{2}=\frac{5}{3}$. Then, for $n_{1}=n_{2}$, the approximation rate is $N^{-\alpha} / 2 d=N^{-1 / 6}$, while, using the asymptotically optimal inter-component distribution, we obtain the rate $N^{-\rho / 2}=N^{-1 / 4.2}=\mathrm{o}\left(N^{-1 / 6}\right)$ as $N \rightarrow \infty$.

In a general setting, numerical procedures can be used to find optimal densities. However, in practice such methods are very computationally demanding. We present a simplification of the expression for the asymptotic constant for one-dimensional components. Furthermore, in this case, we provide the exact formula for the density minimizing the asymptotic constant. For a random field $X \in \mathscr{B}_{l}^{\alpha}(\mathcal{D}, c(\cdot))$, define the integrated local stationarity functions

$$
C_{j}\left(t_{L_{j}}\right):=\int_{[0,1]^{d-1}} c_{j}(\boldsymbol{t}) \mathrm{d} t_{1} \cdots \mathrm{d} t_{L_{j}-1} \mathrm{~d} t_{L_{j}+1} \cdots \mathrm{d} t_{d}, \quad t_{L_{j}} \in[0,1], j=1, \ldots, k .
$$

Moreover, for $0<\beta<2$, let

$$
a_{\beta}:=\frac{2}{(\beta+1)(\beta+2)}-\frac{1}{6} .
$$

Proposition 1. Let $X \in \mathcal{B}_{l}^{\alpha}(\mathcal{D}, c(\cdot))$ be a random field approximated by the $P M I X_{N}\left(X, T_{N}\right)$, where $T_{N}$ is $c R S(h, \pi, l)$. If, for some $j, 1 \leq j \leq k, l_{j}=1$, then, for any regular density $h_{j}(\cdot)$, we have

$$
v_{j}=a_{\alpha_{j}} \int_{0}^{1} C_{j}\left(t_{L_{j}}\right) h_{j}\left(t_{L_{j}}\right)^{-\alpha_{j}} \mathrm{~d} t_{L_{j}} .
$$

The density minimizing $v_{j}$ is given by

$$
h_{j, \text { opt }}\left(t_{L_{j}}\right)=\frac{C_{j}\left(t_{L_{j}}\right)^{\gamma_{j}}}{\int_{0}^{1} C_{j}\left(\tau_{L_{j}}\right)^{\gamma_{j}} \mathrm{~d} \tau_{L_{j}}}, \quad t_{L_{j}} \in[0,1],
$$

where $\gamma_{j}:=1 /\left(1+\alpha_{j}\right)$. Furthermore, for such a chosen density, we obtain

$$
v_{j, \mathrm{opt}}=a_{\alpha_{j}}\left(\int_{0}^{1} C_{j}\left(t_{L_{j}}\right)^{\gamma_{j}} \mathrm{~d} t_{L_{j}}\right)^{1 / \gamma_{j}} .
$$

In the next proposition, we give an upper bound for the approximation error together with expressions for generating densities minimizing this upper bound, called suboptimal densities.

Proposition 2. Let $X \in \mathscr{B}_{l}^{\alpha}(\mathscr{D}, c(\cdot))$ be a random field approximated by the $P M I X_{N}\left(X, T_{N}\right)$, where $T_{N}$ is $c R S(h, \pi, l)$. Then

$$
\left\|X-X_{N}\right\|_{2}^{2} \lesssim \sum_{j=1}^{k} \frac{w_{j}}{n_{j}^{\alpha_{j}}} \quad \text { as } N \rightarrow \infty
$$


where

$$
w_{j}=l_{j}^{1+\alpha_{j} / 2}\left(a_{\alpha_{j}}+\frac{1}{6}\right) \int_{0}^{1} C_{j}\left(t_{L_{j}}\right) h_{j}\left(t_{L_{j}}\right)^{-\alpha_{j}} \mathrm{~d} t_{L_{j}}, \quad j=1, \ldots, k .
$$

The density minimizing $w_{j}$ is given by

$$
h_{j, \text { subopt }}\left(t_{L_{j}}\right)=\frac{C_{j}\left(t_{L_{j}}\right)^{\gamma_{j}}}{\int_{0}^{1} C_{j}\left(\tau_{L_{j}}\right)^{\gamma_{j}} \mathrm{~d} \tau_{L_{j}}}, \quad t_{L_{j}} \in[0,1],
$$

where $\gamma_{j}:=1 /\left(1+\alpha_{j}\right), j=1, \ldots, k$. Furthermore, for such chosen densities, we obtain

$$
w_{j, \text { subopt }}=l_{j}^{1+\alpha_{j} / 2}\left(a_{\alpha_{j}}+\frac{1}{6}\right)\left(\int_{0}^{1} C_{j}\left(t_{L_{j}}\right)^{\gamma_{j}} \mathrm{~d} t_{L_{j}}\right)^{1 / \gamma_{j}}, \quad j=1, \ldots, k .
$$

Now we focus on random fields satisfying the introduced Hölder-type conditions. In this case, we provide results for the uniform mean-squared norm of the approximation error $\left\|X-X_{N}\right\|_{\infty}$. The following proposition provides an upper bound for the accuracy of the PMI for Hölder classes of continuous and continuously differentiable fields.

Proposition 3. Let $X \in \mathcal{C}(D)$ be a random field approximated by the $P M I X_{N}\left(X, T_{N}\right)$, where $T_{N}$ is $c R S(h, \pi, l)$.

(i) If $X \in \mathcal{C}_{l}^{\alpha}(\mathcal{D}, C)$ then, for some positive constants $c_{1}, \ldots, c_{k}$,

$$
\left\|X-X_{N}\right\|_{\infty} \leq \sqrt{C} \sum_{j=1}^{k} \frac{c_{j}}{n_{j}^{\alpha_{j} / 2}} .
$$

(ii) If $X \in \mathcal{C}_{l}^{1, \alpha}(D, C)$ then, for some positive constants $d_{1}, \ldots, d_{k}$,

$$
\left\|X-X_{N}\right\|_{\infty} \leq \sqrt{C} \sum_{j=1}^{k} \frac{d_{j}}{n_{j}^{1+\alpha_{j} / 2}} .
$$

Remark 3. It follows from the proof of Proposition 3 that inequality (12) holds if $c_{j}^{2}=$ $2^{-\alpha_{j}} l_{j}^{1+\alpha_{j} / 2} D_{j}^{\alpha_{j}}, j=1, \ldots, k$, where $D_{j}:=1 / \min _{s \in[0,1]} h_{j}(s), j=1, \ldots, k$. Therefore, the constants depend only on the parameters of the Hölder class and the corresponding sampling design. Similar formulas can be obtained for $d_{1}, \ldots, d_{k}$ in (13).

In addition, we provide the inter-component knot distribution leading to an increased rate of the upper bounds obtained in Proposition 3.

Remark 4. Let $X \in \mathcal{C}(\mathscr{D})$ be a random field approximated by the PMI $X_{N}\left(X, T_{N}\right)$, where $T_{N}$ is $c R S(h, \pi, l)$.

(i) If $X \in \mathcal{C}_{l}^{\alpha}(\mathcal{D}, C)$ and $n_{j} \sim N^{\rho_{0} / \alpha_{j}}, j=1, \ldots, k$, where $\rho_{0}:=\left(\sum_{i=1}^{k} l_{i} / \alpha_{i}\right)^{-1}$, then

$$
\left\|X-X_{N}\right\|_{\infty}=O\left(N^{-\rho_{0} / 2}\right) \quad \text { as } N \rightarrow \infty .
$$

(ii) If $X \in \mathcal{C}_{l}^{1, \boldsymbol{\alpha}}(\mathcal{D}, C)$ and $n_{j} \sim N^{\rho_{1} /\left(2+\alpha_{j}\right)}, j=1, \ldots, k$, where $\rho_{1}:=\left(\sum_{i=1}^{k} l_{i} /(2+\right.$ $\left.\left.\alpha_{i}\right)\right)^{-1}$, then

$$
\left\|X-X_{N}\right\|_{\infty}=O\left(N^{-\rho_{1} / 2}\right) \quad \text { as } N \rightarrow \infty .
$$

The approximation rates obtained in the above remark are optimal in a certain sense, i.e. the rate of convergence cannot be improved in general for random fields satisfying the Höldertype condition (see, e.g. Ritter (2000)). Moreover, these rates correspond to the optimal 
approximation rates for anisotropic Nikolskii-Hölder classes (see, e.g. Yanjie and Yongping (2000)), which are deterministic analogues of the introduced Hölder classes.

\section{Numerical experiments}

In this section, we present some examples illustrating the obtained results. For given knot densities and covariance functions, first the pointwise approximation errors are found analytically. Then numerical integration is used to evaluate the approximation errors on the entire unit hypercube.

In what follows, we use $e_{N}(h, \pi)^{2}=e_{N}(X, h, \pi, l)^{2}$ to denote the IMSE of a PMI approximation of a local stationary field when a cross regular sequence of sampling designs is used. We write $h_{\text {uni }}(\cdot)$, to denote the vector of within-component uniform densities. Analogously, by $\pi_{\text {uni }}(\cdot)$ we denote the uniform inter-dimensional knot distribution, i.e. $n_{1}=\cdots=n_{k}$. Henceforth, we use $\log x$ to denote the natural logarithm of $x$.

Example 4. Let $\mathscr{D}=[0,1]^{3}$ and $X(\boldsymbol{t})=B_{\boldsymbol{\alpha}, \boldsymbol{l}}(\boldsymbol{t})$, where $\boldsymbol{\alpha}=\left(\frac{1}{2}, \frac{3}{2}\right)$ and $\boldsymbol{l}=(1,2)$ (cf. Example 1). Then $X \in \mathcal{B}_{l}^{\alpha}\left([0,1]^{3}, c(\cdot)\right)$, with $c(t)=(1,1), t \in[0,1]^{3}, k=2$, and $\boldsymbol{\alpha}^{*}=\left(\frac{1}{2}, \frac{3}{2}, \frac{3}{2}\right)$. We compare the behaviors of $e_{N}\left(h_{\mathrm{uni}}, \pi_{\mathrm{uni}}\right)$ and $e_{N}\left(h_{\mathrm{uni}}, \pi_{\mathrm{opt}}\right)$, where $\pi_{\mathrm{opt}}$ is given in Theorem 2. Observe that, by using the asymptotically optimal inter-component distribution, we obtain a gain in the rate of approximation. In Figure 1 we present the (fitted) values of the IMSEs $e_{N}\left(h_{\mathrm{uni}}, \pi_{\mathrm{uni}}\right)^{2}$ and $e_{N}\left(h_{\mathrm{uni}}, \pi_{\mathrm{opt}}\right)^{2}$ in a log-log scale. In such a scale, the slopes of fitted lines correspond to the rates of approximation. These plots represent the following asymptotic behavior:

$$
\begin{gathered}
e_{N}\left(h_{\mathrm{uni}}, \pi_{\mathrm{uni}}\right)^{2} \sim 0.3667 N^{-1 / 6}+0.0935 N^{-1 / 2} \sim 0.3667 N^{-1 / 6} \quad \text { as } N \rightarrow \infty, \\
e_{N}\left(h_{\mathrm{uni}}, \pi_{\mathrm{opt}}\right)^{2} \sim 0.4245 N^{-3 / 10} \quad \text { as } N \rightarrow \infty .
\end{gathered}
$$

Example 5. Let $\mathcal{D}=[0,1]^{2}$, and define $Y(\boldsymbol{t})=Y\left(t_{1}, t_{2}\right)$ to be a zero-mean Gaussian field with exponential covariance function, i.e. $\operatorname{cov}(Y(\boldsymbol{t}), Y(\boldsymbol{s}))=\exp (-|\boldsymbol{t}-\boldsymbol{s}|)$. Define $X(\boldsymbol{t})=$ $\left(|\boldsymbol{t}|^{2}+0.1\right)^{-1} Y(\boldsymbol{t}), \boldsymbol{t} \in \mathcal{D}$. Then

$$
\operatorname{cov}(X(t), X(s))=\frac{1}{\left(|t|^{2}+0.1\right)} \frac{1}{\left(|s|^{2}+0.1\right)} \exp (-|t-s|)
$$

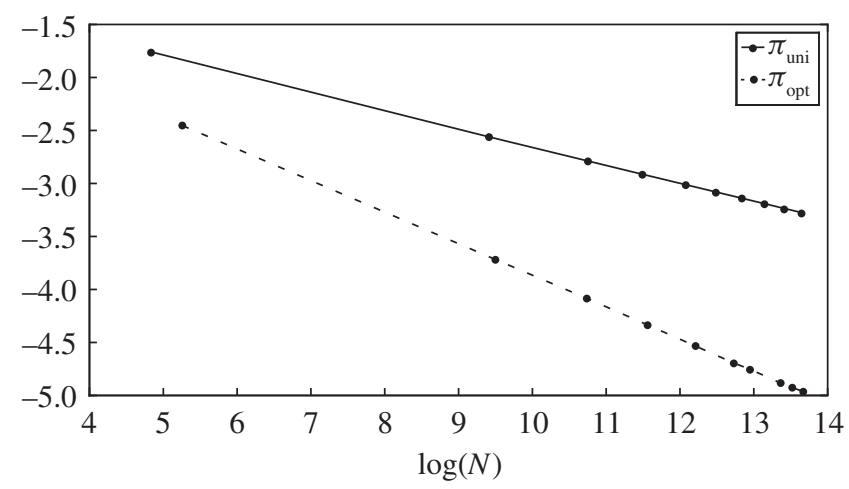

Figure 1: The (fitted) plots of $e_{N}\left(h_{\mathrm{uni}}, \pi_{\mathrm{uni}}\right)^{2}$ (solid line) and $e_{N}\left(h_{\mathrm{uni}}, \pi_{\mathrm{opt}}\right)^{2}$ (dashed line) versus $N$ in a log-log scale. 


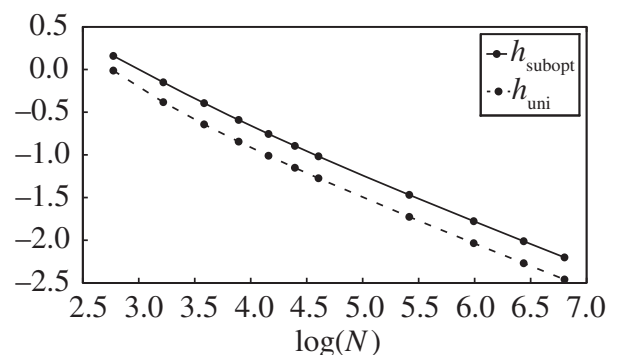

(a)

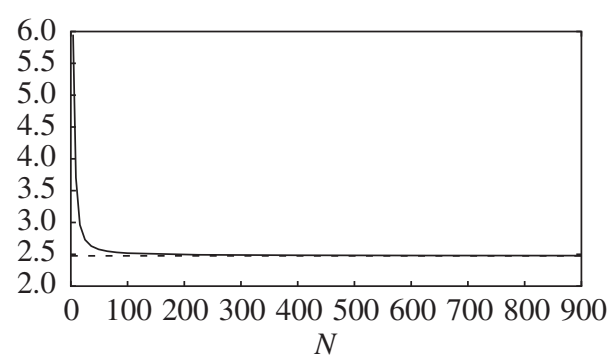

(b)

FIGURE 2: (a) The (fitted) plots of $e_{N}\left(h_{\mathrm{uni}}, \pi_{\mathrm{uni}}\right)^{2}$ (dashed line) and $e_{N}\left(h_{\mathrm{subopt}}, \pi_{\mathrm{uni}}\right)^{2}$ (solid line) versus $N$ in a log-log scale. (b) The convergence of $N^{0.5} e_{N}\left(h_{\text {subopt }}, \pi_{\text {uni }}\right)^{2}$ (solid line) to the asymptotic constant (dashed line).

and $X \in \mathcal{B}_{l}^{\boldsymbol{\alpha}}\left([0,1]^{2}, c(\cdot)\right)$ with $c(\boldsymbol{t})=c_{1}(\boldsymbol{t})=2 /\left(|\boldsymbol{t}|^{2}+0.1\right)^{2}, \boldsymbol{t} \in[0,1]^{2}, \boldsymbol{\alpha}=1$, $\boldsymbol{\alpha}^{*}=(1,1), \boldsymbol{l}=2$, and $k=1$. The field has one component; hence, the uniform interdimensional knot distribution is used. Theorem 2 provides the formula for the suboptimal within-component density. In Figure 2(a) we present the (fitted) values of the IMSEs $e_{N}\left(h_{\text {uni }}, \pi_{\text {uni }}\right)^{2}$ and $e_{N}\left(h_{\text {subopt }}, \pi_{\text {uni }}\right)^{2}$. In Figure 2(b) we present the convergence of the scaled squared approximation error $N^{0.5} e_{N}\left(h_{\text {subopt }}, \pi_{\text {uni }}\right)^{2}$ to the asymptotic constant obtained in Theorem 1. Note that utilizing the suboptimal within-component density leads to a significant reduction of the asymptotic constant, as compared to the uniform within-component knot distribution.

\section{Proofs}

Proof of Lemma 1. We start by observing that, by the definition of the interpolator,

$$
\begin{aligned}
e(\boldsymbol{\tau}, \boldsymbol{r}, \boldsymbol{t})^{2}= & \mathbb{E}(X(\boldsymbol{t})-I(X, \boldsymbol{\tau}, \boldsymbol{r}, t))^{2} \\
= & \mathbb{E}\left(\mathbb{E}_{\boldsymbol{\eta}}(X(\boldsymbol{\tau}+\boldsymbol{r} * \boldsymbol{\eta})-X(\boldsymbol{t}))\right)^{2} \\
= & \mathbb{E}_{\boldsymbol{\eta}, \boldsymbol{\xi}} \mathbb{E}((X(\boldsymbol{\tau}+\boldsymbol{r} * \boldsymbol{\eta})-X(\boldsymbol{t}))(X(\boldsymbol{\tau}+\boldsymbol{r} * \boldsymbol{\xi})-X(\boldsymbol{t}))) \\
= & \frac{1}{2} \mathbb{E}_{\boldsymbol{\eta}, \boldsymbol{\xi}} \mathbb{E}\left((X(\boldsymbol{\tau}+\boldsymbol{r} * \boldsymbol{\eta})-X(\boldsymbol{t}))^{2}+(X(\boldsymbol{\tau}+\boldsymbol{r} * \boldsymbol{\xi})-X(\boldsymbol{t}))^{2}\right. \\
& \left.\quad-(X(\boldsymbol{\tau}+\boldsymbol{r} * \boldsymbol{\eta})-X(\boldsymbol{\tau}+\boldsymbol{r} * \boldsymbol{\xi}))^{2}\right),
\end{aligned}
$$

where $\xi$ is an independent copy of $\eta$. Furthermore, property (2) together with the uniform continuity and positiveness of the local stationarity functions $c_{1}(\cdot), \ldots, c_{k}(\cdot)$ imply that

$$
\begin{array}{r}
e(\boldsymbol{\tau}, \boldsymbol{r}, \boldsymbol{t})^{2}=\frac{1}{2}\left(\sum _ { j = 1 } ^ { k } c _ { j } ( \boldsymbol { \tau } ) \mathbb { E } _ { \boldsymbol { \eta } , \boldsymbol { \xi } } \left(\left|\boldsymbol{r}^{j} *\left(\boldsymbol{\eta}^{j}-\boldsymbol{s}^{j}\right)\right|^{\alpha_{j}}+\left|\boldsymbol{r}^{j} *\left(\boldsymbol{\xi}^{j}-\boldsymbol{s}^{j}\right)\right|^{\alpha_{j}}\right.\right. \\
\left.\left.-\left|\boldsymbol{r}^{j} *\left(\boldsymbol{\eta}^{j}-\boldsymbol{\xi}^{j}\right)\right|^{\alpha_{j}}\right)\right)(1+\varepsilon(\boldsymbol{\tau}, \boldsymbol{r})),
\end{array}
$$

where $s$ is defined by (6) and $\varepsilon(\boldsymbol{r}):=\max \{|\varepsilon(\boldsymbol{\tau}, \boldsymbol{r})|, \boldsymbol{\tau} \in \mathcal{D}\}=\mathrm{o}(1)$ as $|\boldsymbol{r}| \rightarrow 0$ (cf. the proof of Theorem 1 of Seleznjev (2000)). Define

$$
\begin{aligned}
C_{\alpha_{j}, l_{j}}\left(\boldsymbol{s}^{j}, \boldsymbol{r}^{j}\right) & :=\frac{1}{2} \mathbb{E}_{\boldsymbol{\eta}, \boldsymbol{\xi}}\left(\left|\boldsymbol{r}^{j} *\left(\boldsymbol{\eta}^{j}-\boldsymbol{s}^{j}\right)\right|^{\alpha_{j}}+\left|\boldsymbol{r}^{j} *\left(\boldsymbol{\xi}^{j}-\boldsymbol{s}^{j}\right)\right|^{\alpha_{j}}-\left|\boldsymbol{r}^{j} *\left(\boldsymbol{\eta}^{j}-\boldsymbol{\xi}^{j}\right)\right|^{\alpha_{j}}\right) \\
& =\left\|B_{\alpha_{j}, l_{j}}\left(\boldsymbol{r}^{j} * \boldsymbol{s}^{j}\right)-\mathbb{E}_{\boldsymbol{\eta}} B_{\alpha_{j}, l_{j}}\left(\boldsymbol{r}^{j} * \boldsymbol{\eta}^{j}\right)\right\|_{2}^{2}, \quad j=1, \ldots, k .
\end{aligned}
$$


Then by the definition we obtain

$$
\begin{aligned}
e(\boldsymbol{\tau}, \boldsymbol{r})^{2} & =\int_{\mathcal{K}} e(\boldsymbol{\tau}, \boldsymbol{r}, \boldsymbol{t})^{2} \mathrm{~d} \boldsymbol{t} \\
& =\left(\int_{\mathcal{K}} \sum_{j=1}^{k} c_{j}(\boldsymbol{\tau}) C_{\alpha_{j}, l_{j}}\left(\boldsymbol{s}^{j} ; \boldsymbol{r}^{j}\right) \mathrm{d} \boldsymbol{t}\right)(1+\mathrm{o}(1)) \\
& =\left(\sum_{j=1}^{k} c_{j}(\boldsymbol{\tau}) \int_{\mathcal{D}^{j}} C_{\alpha_{j}, l_{j}}\left(\boldsymbol{s}^{j} ; \boldsymbol{r}^{j}\right) \mathrm{d} \boldsymbol{s}^{j} V(\mathcal{K})\right)(1+\mathrm{o}(1)) \\
& =\left(\sum_{j=1}^{k} n_{j}^{-\alpha_{j}} \sum_{\boldsymbol{i} \in \boldsymbol{I}} c_{j}\left(\boldsymbol{t}_{i}\right) b_{\alpha_{j}, l_{j}}\left(\boldsymbol{r}^{j}\right)\right) V(\mathcal{K})(1+\mathrm{o}(1)) \quad \text { as }|\boldsymbol{r}| \rightarrow 0
\end{aligned}
$$

with term o(1) being uniformly small over all $\tau \in \mathscr{D}$. This completes the proof.

Proof of Theorem 1. For any hyperrectangle $\mathscr{D}_{\boldsymbol{i}}, \boldsymbol{i} \in \boldsymbol{I}$, where $\boldsymbol{I}:=\left\{\boldsymbol{i}=\left(i_{1}, \ldots, i_{d}\right)\right.$, $\left.0 \leq i_{k} \leq n_{k}^{*}-1, k=1, \ldots, d\right\}$, it follows from the definition and the mean (integral) value theorem that its diagonal $\boldsymbol{r}_{i}$ satisfies

$$
\boldsymbol{r}_{\boldsymbol{i}}=\left(\frac{1}{n_{1}^{*} h_{1}^{*}\left(w_{1, i_{1}}\right)}, \ldots, \frac{1}{n_{d}^{*} h_{d}^{*}\left(w_{d, i_{d}}\right)}\right)
$$

for appropriate $w_{j, i_{j}} \in\left[t_{j, i_{j}}, t_{j, i_{j}+1}\right], j=1, \ldots, d$. Define $w_{i}:=\left(w_{1, i_{1}}, \ldots, w_{d, i_{d}}\right)$. Now the definition of $c R S(h, \pi, l)$ implies that, for the $j$ th component of the diagonal $\boldsymbol{r}_{\boldsymbol{i}}$, we have

$$
\boldsymbol{r}_{\boldsymbol{i}}^{j}=\frac{1}{n_{j}}\left(\frac{1}{h_{j}\left(w_{L_{j-1}+1, i_{L_{j-1}+1}}\right)}, \ldots, \frac{1}{h_{j}\left(w_{L_{j}, i_{L_{j}}}\right)}\right)=\frac{1}{n_{j}} H_{j}\left(\boldsymbol{w}_{\boldsymbol{i}}^{j}\right), \quad j=1, \ldots, k,
$$

where $H_{j}\left(\boldsymbol{t}^{j}\right):=\left(1 / h_{j}\left(t_{L_{j-1}+1}\right), \ldots, 1 / h_{j}\left(t_{L_{j}}\right)\right), j=1, \ldots, k$. Applying Lemma 1 for hyperrectangles $\left\{\mathscr{D}_{i}\right\}$ and the uniform continuity of $h(\cdot)$ we obtain

$$
\begin{aligned}
e_{N}^{2} & =\left(\sum_{j=1}^{k} \sum_{\boldsymbol{i} \in \boldsymbol{I}} c_{j}\left(\boldsymbol{t}_{i}\right) b_{\alpha_{j}, l_{j}}\left(\boldsymbol{r}_{\boldsymbol{i}}\right) V\left(\mathscr{D}_{\boldsymbol{i}}\right)\right)(1+\mathrm{o}(1)) \\
& =\left(\sum_{j=1}^{k} n_{j}^{-\alpha_{j}} \sum_{\boldsymbol{i} \in \boldsymbol{I}} c_{j}\left(\boldsymbol{t}_{i}\right) b_{\alpha_{j}, l_{j}}\left(H_{j}\left(\boldsymbol{w}_{\boldsymbol{i}}^{j}\right)\right) V\left(\mathscr{D}_{\boldsymbol{i}}\right)\right)(1+\mathrm{o}(1)) \\
& =\left(\sum_{j=1}^{k} n_{j}^{-\alpha_{j}} \sum_{\boldsymbol{i} \in \boldsymbol{I}} c_{j}\left(\boldsymbol{t}_{i}\right) b_{\alpha_{j}, l_{j}}\left(H_{j}\left(\boldsymbol{t}_{\boldsymbol{i}}^{j}\right)\right) V\left(\mathscr{D}_{\boldsymbol{i}}\right)\right)(1+\mathrm{o}(1)) \quad \text { as } N \rightarrow \infty .
\end{aligned}
$$

Now the Riemann integrability of the functions $t \rightarrow c_{j}(\boldsymbol{t}) b_{\alpha_{j}, l_{j}}\left(H_{j}\left(\boldsymbol{t}^{j}\right)\right), j=1, \ldots, k$, gives, as $N \rightarrow \infty$,

$$
e_{N}^{2}=\left(\sum_{j=1}^{k} n_{j}^{-\alpha_{j}} \int_{\mathcal{D}} c_{j}(\boldsymbol{t}) b_{\alpha_{j}, l_{j}}\left(H_{j}\left(\boldsymbol{t}^{j}\right)\right) \mathrm{d} \boldsymbol{t}\right)(1+\mathrm{o}(1))=\left(\sum_{j=1}^{k} \frac{v_{j}}{n_{j}^{\alpha_{j}}}\right)(1+\mathrm{o}(1)) .
$$

Note that, for any $\boldsymbol{u} \in \mathbb{R}_{+}^{m}$, we have $b_{\beta, m}(\boldsymbol{u})>0$; otherwise, the fractional Brownian field is degenerate (cf. Seleznjev (2000)). Consequently, $v_{j}>0, j=1, \ldots, k$. This completes the proof. 
Proof of Theorem 2. By the inequality for the arithmetic and geometric means,

$$
\frac{1}{k} \sum_{j=1}^{k} \frac{v_{j}}{n_{j}^{\alpha_{j}}} \geq\left(\prod_{j=1}^{k} \frac{v_{j}}{n_{j}^{\alpha_{j}}}\right)^{1 / k}
$$

with equality if and only if $v^{-1}=v_{j} / n_{j}^{\alpha_{j}}, j=1, \ldots, k$. Hence, the equality is attained for $\tilde{n}_{j}=\left(v v_{j}\right)^{1 / \alpha_{j}}, j=1, \ldots, k$. Let

$$
n_{j}=\left\lceil\tilde{n}_{j}\right\rceil \sim\left(\nu v_{j}\right)^{1 / \alpha_{j}} \quad \text { as } N \rightarrow \infty .
$$

The total number of observations satisfies

$$
N=\left(n_{1}^{*}+1\right) \cdots\left(n_{d}^{*}+1\right) \sim \prod_{i=1}^{d} n_{i}^{*}=\prod_{j=1}^{k} n_{j}^{l_{j}}=M \quad \text { as } N \rightarrow \infty .
$$

This implies that, for the asymptotically optimal inter-component knot distribution, we have

$$
N \sim M \sim v^{1 / \rho} \prod_{j=1}^{k} v_{j}^{l_{j} / \alpha_{j}},
$$

and, therefore, $v \sim N^{\rho} \kappa^{-\rho}$ as $N \rightarrow \infty$. By (15), the asymptotically optimal inter-component knot distribution is

$$
n_{j} \sim \frac{N^{\rho / \alpha_{j}} v_{j}^{1 / \alpha_{j}}}{\kappa^{\rho / \alpha_{j}}} \quad \text { as } N \rightarrow \infty, j=1, \ldots, k .
$$

Moreover, with such a chosen knot distribution, the equality in (9) is attained asymptotically. This completes the proof.

Proof of Proposition 1. The proof is a straightforward implication of the assumptions and (14). The exact constant and the expression for the optimal density are due to Seleznjev (2000).

Proof of Proposition 2. Consider the random variable $\eta \in \mathrm{Be}(s)$. For any $0<\beta<2$, we define

$$
A_{\beta}(s):=\mathbb{E}_{\eta}|\eta-s|^{\beta}=(1-s)^{\beta} s+(1-s) s^{\beta}
$$

and

$$
A_{\beta}:=\int_{0}^{1} A_{\beta}(s) \mathrm{d} s=\frac{2}{(\beta+1)(\beta+2)}=a_{\beta}+\frac{1}{6},
$$

where $a_{\beta}$ is defined by (11). It follows from (7) and (8) that, for any $\boldsymbol{u} \in \mathbb{R}_{+}^{m}$,

$$
\begin{aligned}
b_{\beta, m}(\boldsymbol{u}) & =\int_{[0,1]^{m}}\left\|B_{\beta, m}(\boldsymbol{u} * \boldsymbol{s})-\mathbb{E}_{\boldsymbol{\eta}} B_{\beta, m}(\boldsymbol{u} * \boldsymbol{\eta})\right\|^{2} \mathrm{~d} \boldsymbol{s} \\
& \leq \int_{[0,1]^{m}} \mathbb{E}_{\boldsymbol{\eta}}|\boldsymbol{u} *(\boldsymbol{s}-\boldsymbol{\eta})|^{\beta} \mathrm{d} \boldsymbol{s} \\
& \leq m^{\beta / 2} \sum_{i=1}^{m} u_{i}^{\beta} \int_{[0,1]^{m}} A_{\beta}\left(s_{i}\right) \mathrm{d} \boldsymbol{s} \\
& =m^{\beta / 2} A_{\beta} \sum_{i=1}^{m} u_{i}^{\beta},
\end{aligned}
$$


where the second inequality is due to the following property for any nonnegative numbers $a_{1}, \ldots, a_{k}$ and any $\alpha \in \mathbb{R}_{+}$:

$$
\left(\sum_{i=1}^{k} a_{i}\right)^{\alpha} \leq k^{\alpha} \sum_{i=1}^{k} a_{i}^{\alpha}
$$

Applying (16) yields

$$
\begin{aligned}
v_{j} & =\int_{\mathscr{D}} c_{j}(\boldsymbol{t}) b_{\alpha_{j}, l_{j}}\left(H_{j}\left(\boldsymbol{t}^{j}\right)\right) \mathrm{d} \boldsymbol{t} \\
& \leq l_{j}^{\alpha_{j} / 2} A_{\alpha_{j}} \sum_{m=L_{j-1}+1}^{L_{j}} \int_{\mathscr{D}} c_{j}(\boldsymbol{t}) h_{j}\left(t_{m}\right)^{-\alpha_{j}} \mathrm{~d} \boldsymbol{t} \\
& =l_{j}^{1+\alpha_{j} / 2} A_{\alpha_{j}} \int_{0}^{1} C_{j}\left(t_{L_{j}}\right) h_{j}\left(t_{L_{j}}\right)^{-\alpha_{j}} \mathrm{~d} t_{L_{j}}, \quad j=1, \ldots, k .
\end{aligned}
$$

The assertion now follows by Theorem 1 . This completes the proof.

Proof of Proposition 3. We start by proving (i). Let $X \in \mathcal{C}_{l}^{\alpha}\left([0,1]^{d}, C\right)$, and consider $\boldsymbol{t} \in \mathscr{D}_{\boldsymbol{i}}, \boldsymbol{i} \in \boldsymbol{I}$. Applying (8) and Hölder's condition (1) we obtain, for $\boldsymbol{t}=\boldsymbol{t}_{\boldsymbol{i}}+\boldsymbol{r}_{\boldsymbol{i}} * \boldsymbol{s}$,

$$
\begin{aligned}
& e_{N}(\boldsymbol{t})^{2}=\mathbb{E}\left(\mathbb{E}_{\boldsymbol{\eta}}\left(X(\boldsymbol{t})-X\left(\boldsymbol{t}_{\boldsymbol{i}}+\boldsymbol{r}_{\boldsymbol{i}} * \boldsymbol{\eta}\right)\right)\right)^{2} \\
& \leq \mathbb{E}_{\eta}\left\|X(\boldsymbol{t})-X\left(\boldsymbol{t}_{\boldsymbol{i}}+\boldsymbol{r}_{\boldsymbol{i}} * \boldsymbol{\eta}\right)\right\|^{2} \\
& \leq C \mathbb{E}_{\eta}\left|\boldsymbol{r}_{\boldsymbol{i}} * \eta\right|_{\alpha} \\
& =C \mathbb{E}_{\boldsymbol{\eta}} \sum_{j=1}^{k}\left|\boldsymbol{r}_{i}^{j} * \eta^{j}\right|^{\alpha_{j}} \\
& \leq C \sum_{j=1}^{k} l_{j}^{\alpha_{j} / 2} \sum_{m=L_{j-1}+1}^{L_{j}} r_{i, m} A_{\alpha_{j}}(s),
\end{aligned}
$$

where the last inequality follows from (17). Furthermore, since $\max _{s \in[0,1]} A_{\alpha_{j}}(s)=2^{-\alpha_{j}}$, we obtain

$$
e_{N}(\boldsymbol{t})^{2} \leq \sum_{j=1}^{k} 2^{-\alpha_{j}} l_{j}^{\alpha_{j} / 2} \sum_{m=L_{j-1}+1}^{L_{j}} r_{i, m}^{\alpha_{j}} .
$$

By the regularity of the generating densities, we have $r_{i, m} \leq 1 /\left(n_{m}^{*} \min _{s \in[0,1]} h_{m}^{*}(s)\right), \boldsymbol{i} \in \boldsymbol{I}$, $m=1, \ldots, d$. Moreover, the definition of $c R S(h, \pi, l)$ implies a uniform bound for the squared approximation accuracy

$$
\left\|X-X_{N}\right\|_{\infty}^{2}=\max _{\boldsymbol{t} \in \mathcal{D}} e_{N}^{2}(\boldsymbol{t}) \leq C \sum_{j=1}^{k} 2^{-\alpha_{j}} l_{j}^{1+\alpha_{j} / 2} \frac{D_{j}^{\alpha_{j}}}{n_{j}^{\alpha_{j}}}
$$

with $D_{j}=1 / \min _{s \in[0,1]} h_{j}(s), j=1, \ldots, k$. Finally, we obtain the required assertion

$$
\left\|X-X_{N}\right\|_{\infty} \leq \sqrt{C} \sum_{j=1}^{k} \frac{c_{j}}{n_{j}^{\alpha_{j} / 2}}
$$

where $c_{j}^{2}:=2^{-\alpha_{j}} l_{j}^{1+\alpha_{j} / 2} D_{j}^{\alpha_{j}}>0, j=1, \ldots, k$. 
For the smooth case (ii), we use the multivariate Taylor formula to obtain the following representation of the deviation field for $\boldsymbol{t} \in \mathscr{D}_{i}, \boldsymbol{t}=\boldsymbol{t}_{i}+\boldsymbol{s} * \boldsymbol{r}_{i}$ :

$$
\delta_{n}(\boldsymbol{t}):=X(\boldsymbol{t})-X_{N}(\boldsymbol{t})=\mathbb{E}_{\boldsymbol{\eta}}\left(\int_{0}^{1} \sum_{j=1}^{d} X_{j}^{\prime}\left(\boldsymbol{t}_{i}+u \boldsymbol{r}_{i} *(\boldsymbol{\eta}-\boldsymbol{s})\right) r_{i, j}\left(\eta_{j}-s_{j}\right) \mathrm{d} u\right) .
$$

Here $\eta=\left(\eta_{1}, \ldots, \eta_{d}\right)$ and $\eta_{1}, \ldots, \eta_{d}$ are independent Bernoulli random variables, $\eta_{j} \in$ $\operatorname{Be}\left(s_{j}\right), j=1, \ldots, d$. Introducing an auxiliary uniform random variable $U \in \mathcal{U}(0,1)$ we obtain

$$
\begin{gathered}
\delta_{n}(\boldsymbol{t})=\sum_{j=1}^{d} \mathbb{E}_{\boldsymbol{\eta}, U}\left(X_{j}^{\prime}\left(\boldsymbol{t}_{\boldsymbol{i}}+U(\boldsymbol{\eta}-\boldsymbol{s}) * \boldsymbol{r}_{\boldsymbol{i}}\right) r_{\boldsymbol{i}, j}\left(\eta_{j}-s_{j}\right)\right) \\
=\sum_{j=1}^{d} \mathbb{E}_{\boldsymbol{\eta}, U}\left(X _ { j } ^ { \prime } \left(t_{\boldsymbol{i}, 1}+U\left(\eta_{1}-s_{1}\right) r_{\boldsymbol{i}, 1}, \ldots, t_{\boldsymbol{i}, j}+U\left(\eta_{j}-s_{j}\right) r_{\boldsymbol{i}, j},\right.\right. \\
\left.\ldots, t_{\boldsymbol{i}, d}+U\left(\eta_{d}-s_{d}\right) r_{\boldsymbol{i}, d}\right) \\
-X_{j}^{\prime}\left(t_{\boldsymbol{i}, 1}+U\left(\eta_{1}-s_{1}\right) r_{\boldsymbol{i}, 1}, \ldots, t_{\boldsymbol{i}, j}\right. \\
\left.\left.\ldots, t_{i, d}+U\left(\eta_{d}-s_{d}\right) r_{\boldsymbol{i}, d}\right)\right)\left(\eta_{j}-s_{j}\right),
\end{gathered}
$$

since, for any $j=1, \ldots, d$,

$$
\begin{aligned}
& \mathbb{E}_{\boldsymbol{\eta}}\left(X_{j}^{\prime}\left(t_{i, 1}+U\left(\eta_{1}-s_{1}\right) r_{i, 1}, \ldots, t_{i, j}, \ldots, t_{i, d}+U\left(\eta_{d}-s_{d}\right) r_{i, d}\right)\left(\eta_{j}-s_{j}\right)\right) \\
& \quad=\mathbb{E}_{\boldsymbol{\eta}}\left(X_{j}^{\prime}\left(t_{i, 1}+U\left(\eta_{1}-s_{1}\right) r_{i, 1}, \ldots, t_{i, j}, \ldots, t_{i, d}+U\left(\eta_{d}-s_{d}\right) r_{i, d}\right) \mathbb{E}_{\eta_{j}}\left(\eta_{j}-s_{j}\right)\right) \\
& \quad=0 .
\end{aligned}
$$

The triangle inequality and condition (4) imply that

$$
e_{N}(\boldsymbol{t}) \leq \sum_{j=1}^{d} \sqrt{C} V_{j} r_{i, j}^{1+\alpha_{j} / 2}
$$

for some positive constants $V_{j}, j=1, \ldots, d$. Analogously to (i), the required assertion follows from the regularity of the generating densities and the definition of $c R S(h, \pi, l)$. This completes the proof.

\section{Acknowledgements}

The second author was partly supported by the Swedish Research Council grant 2009-4489 and the project 'Digital Zoo' funded by the European Regional Development Fund.

\section{References}

Abramowicz, K. and Seleznjev, O. (2011). Spline approximation of a random process with singularity. J. Statist. Planning Inf. 141, 1333-1342.

AdLeR, R. AND TAYLOR, J. (2007). Random Fields and Geometry. Springer, New York.

BenHenni, K. (2001). Reconstruction of a stationary spatial process from a systematic sampling. In Selected Proceedings of the Symposium on Inference for Stochastic Processes (IMS Lecture Notes Monogr. Ser. 37), Institute of Mathematical Statistics, Beachwood, OH, pp. 271-279.

Berman, S. M. (1974). Sojourns and extremes of Gaussian processes. Ann. Prob. 2, 999-1026. 
Brouste, A., Istas, J. And Lambert-Lacroix, S. (2007). On fractional Gaussian random fields simulations. J. Statist. Software 23, 1-23.

Christakos, G. (1992). Random Field Models in Earth Sciences. Academic Press, London.

De Boor, C., Gout, C., Kunoth, A. And Rabut, C. (2008). Multivariate approximation: theory and applications. An overview. Numer. Algorithms 48, 1-9.

Hüsler, J., Piterbarg, V. And Seleznjev, O. (2003). On convergence of the uniform norms for Gaussian processes and linear approximation problems. Ann. Appl. Prob. 13, 1615-1653.

Kuo, F. Y., WAsILKowski, G. W. AND WoźNIAKOWSKI, H. (2009). On the power of standard information for multivariate approximation in the worst case setting. J. Approximation Theory 158, 97-125.

Lancaster, P. and Šalkauskas, K. (1986). Curve and Surface Fitting. An Introduction. Academic Press, London.

LifShits, M. A. AND ZANI, M. (2008). Approximation complexity of additive random fields. J. Complexity 24, $362-379$.

Müller-Gronbach, T. (1998). Hyperbolic cross designs for approximation of random fields. J. Statist. Planning Inf. 66, 321-344.

Müller-Gronbach, T. And Schwabe, R. (1996). On optimal allocations for estimating the surface of a random field. Metrika 44, 239-258.

NikolskiI, S. M. (1975). Approximation of Functions of Several Variables and Imbedding Theorems. Springer, Berlin.

Pratt, W. K. (2007). Digital Image Processing: PIKS Scientific Inside. John Wiley, New York.

RitTer, K. (2000). Average-Case Analysis of Numerical Problems. Springer, Berlin.

RitTer, K., WasilkowsKi, G. W. AND WoźniaKowski, H. (1995). Multivariate integration and approximation for random fields satisfying Sacks-Ylvisaker conditions. Ann. Appl. Prob. 5, 518-540.

SACKS, J. ANd YlvisaKer, D. (1966). Designs for regression problems with correlated errors. Ann. Math. Statist. 37, 66-89.

SelezNJeV, O. (1996). Large deviations in the piecewise linear approximation of Gaussian processes with stationary increments. Adv. Appl. Prob. 28, 481-499.

Seleznjev, O. (2000). Spline approximation of random processes and design problems. J. Statist. Planning Inf. 84, 249-262.

STEIN, M. (1999). Interpolation of Spatial Data. Springer, New York.

Su, Y. (1997). Estimation of random fields by piecewise constant estimators. Stoch. Process. Appl. 71, 145-163.

Su, Y. and Cambanis, S. (1993). Sampling designs for estimation of a random process. Stoch. Process. Appl. 46, 47-89.

YANJIE, J. AND YoNGPING, L. (2000). Average widths and optimal recovery of multivariate Besov classes in $L_{p}\left(R^{d}\right)$. J. Approximation Theory 102, 155-170.

ZhANG, X. AND WiCKer, S. B. (2005). On the optimal distribution of sensors in a random field. ACM Trans. Sensor Networks 1, 301-306. 